\title{
The efficacy and safety of nivolumab in previously treated advanced non-small-cell lung cancer: a meta-analysis of prospective clinical trials
}

This article was published in the following Dove Press journal:

OncoTargets and Therapy

23 September 2016

Number of times this article has been viewed

\author{
Jiaxing Huang ${ }^{1,2, *}$ \\ Yaxiong Zhang ${ }^{1,2, *}$ \\ Jin Sheng ${ }^{1,2, *}$ \\ Hongyu Zhang ${ }^{3}$ \\ Wenfeng Fang ${ }^{1,2}$ \\ Jianhua Zhan ${ }^{1,2}$ \\ Ting Zhou ${ }^{1,2}$ \\ Ying Chen ${ }^{1,2}$ \\ Lin Liu ${ }^{1,2}$ \\ Li Zhang ${ }^{1,2}$ \\ 'Department of Medical Oncology, \\ Sun Yat-Sen University Cancer Center, \\ ${ }^{2}$ State Key Laboratory of Oncology \\ in South China, ${ }^{3}$ Department of \\ Medical Oncology, the Fifth Affiliated \\ Hospital of Sun Yat-Sen University, \\ Zhuhai, Guangdong, People's Republic \\ of China
}

*These authors contributed equally to this work
Correspondence: Li Zhang Department of Medical Oncology, Sun Yat-Sen University Cancer Center, 65I Dongfeng East Road, Guangzhou, China, 510060, Guangzhou, People's Republic of China Email zhangli@sysucc.org.cn
Background: Nivolumab (BMS-936558/ONO-4538) was the first monoclonal antibody targeting programmed death (PD)-1. So far, a number of clinical trials on nivolumab have showed satisfactory efficacy in treating non-small-cell lung cancer (NSCLC). Herein, we present a meta-analysis evaluating the efficacy and safety of nivolumab for previously treated advanced NSCLC patients.

Methods: Electronic databases were searched for eligible literature. Data of objective response rate (ORR), disease control rate, overall survival, progression-free survival, and adverse effects (AEs) were extracted and pooled. Outcomes analyzed and presented in this study were according to the original data from nivolumab $3 \mathrm{mg} / \mathrm{kg}$.

Results: In general, nine trials with 817 patients were included in this meta-analysis. The pooled ORR, disease control rate, 1-year overall survival rate, and 1-year progression-free survival rate were $20 \%$ (95\% confidence interval [CI]: 17\%-23\%), 36\% (95\% CI: $22 \%-51 \%$ ), $47 \%$ (95\% CI: 40\%-53\%), 21\% (95\% CI: 18\%-24\%), respectively. In addition, the rate of grade 3-4 AEs was only 8\% (95\% CI: 6\%-12\%). Subgroup analysis showed no significant difference in terms of ORR between squamous and non-squamous NSCLC (odds ratio 1.23, 95\% CI: $0.63-2.39, P=0.51$ ). However, significantly greater ORR was presented in programmed cell death ligand 1 (PD-L1) positive cohort (ORR 31\%, 95\% CI: 24\%-38\%), compared to PD-L1 negative cohort (ORR 12\%, 95\% CI: 9\%-17\%). The odds ratio for objective response to nivolumab in PD-L1 positive cases relative to negative cases was 3.08 (95\% CI: $1.87-5.08, P<0.0001$ ).

Conclusion: In conclusion, nivolumab is a promising second-line agent for previously treated advanced NSCLC with manageable AEs. Both squamous and non-squamous NSCLC patients showed similar efficacy. In addition, patients with positive PD-L1 expression had better response from nivolumab.

Microabstract: We present a meta-analysis evaluating the efficacy and safety of nivolumab for previously treated advanced NSCLC patients. In our study, nivolumab is a promising secondline agent for previously treated advanced NSCLC with manageable AEs. Both squamous and non-squamous NSCLC patients showed similar efficacy. In addition, patients with positive PD-L1 expression had better response from nivolumab.

Keywords: nivolumab, PD-1, non-small-cell lung cancer (NSCLC), meta-analysis

\section{Introduction}

Lung cancer is one of the most common malignancies and the leading cause of cancer-related deaths around the world. ${ }^{1}$ Approximately $85 \%$ of patients have nonsmall-cell lung cancer (NSCLC), and about $80 \%$ cases were diagnosed at an advanced stage. ${ }^{2,3}$ Moreover, the prognosis and overall survival (OS) rate of patients with 
advanced NSCLC are very low. ${ }^{4}$ Surgery, chemotherapy, and radiotherapy were the three primary treatment modalities for NSCLC in the past. In the past decade, targeted therapies, such as epidermal growth factor receptor and anaplastic lymphoma receptor tyrosine kinase inhibitors, have helped control tumor growth effectively for patients harboring specific genetic abnormalities. However, a large proportion of patients have limited benefits due to lack of driver mutation, especially for patients with advanced squamous NSCLC. ${ }^{5-8}$ Nevertheless, for patients whose disease worsens after initial treatment, the second-line docetaxel regimen offers modest benefit. What is more, the AEs of docetaxel regimen are always hard to bear., ${ }^{9,10}$

In these special cases, immune checkpoint inhibition therapy serves as a new therapeutic approach for patients with previously advanced treated NSCLC, which takes effect by restoring the immune system's capacity to recognize and eradicate tumors. ${ }^{11}$ The programmed death (PD)-1 receptor expressed on activated T-cells is engaged by the tumor-expressed ligands PD-L1 (B7-H1) and PD-L2 (B7-H1) to downregulate T-cell activation and promote tumor cells to escape recognition and elimination by the immune system. ${ }^{12}$ Nivolumab (BMS-936558) is a fully human IgG4 PD-1 immune-checkpoint-antibody that disrupts T-cell activation and proliferation and restores antitumor immunity. ${ }^{13-16}$ Recently, a Phase III randomized clinical trial (Checkmate017) ${ }^{17}$ of nivolumab for patients with previously treated advanced squamous NSCLC demonstrated that compared to standard second-line docetaxel regimen, nivolumab significantly gained the better median OS (9 months versus 6 months), nearly doubling the 1 -year survival rate (42\% versus $24 \%$ ). In addition, another Phase III randomized clinical trial (Checkmate057) ${ }^{18}$ for patients with non-squamous NSCLC worsened after platinum-based doublet chemotherapy revealed that compared to standard chemotherapy, nivolumab significantly prolonged the median survival (12.2 months versus 9.4 months). ${ }^{17-19}$ Hence, nivolumab was approved by US Food and Drug Administration for the treatment of squamous as well as non-squamous lung cancer that progressed after platinum-based doublet chemotherapy. ${ }^{19}$ However, these two trials cannot characterize subgroups of patients whose disease progresses early and who may benefit most from nivolumab, and more reliable data of safety of nivolumab are warranted. Beyond that, there have been several trials assessing the efficacy of previously treated advanced NSCLC with nivolumab. ${ }^{13,20-25}$ Moreover, in view of the limitation that different doses within the different phase clinical trials which could display the various efficacy, it is important and necessary to conduct a timely summarization at the same efficacious dose of $3 \mathrm{mg} / \mathrm{kg}$. Recently, several studies demonstrated that the dose $(3 \mathrm{mg} / \mathrm{kg})$ was a preferential dosage of nivolumab. ${ }^{13,20}$ Thus, we performed this meta-analysis systematically combining data from published clinical trials to evaluate the efficacy and safety of nivolumab at the efficacious dose of $3 \mathrm{mg} / \mathrm{kg}$ in previously treated advanced NSCLC.

\section{Materials and methods Literature search}

The relevant data were rigorous searched to identify clinical trials from Embase, PubMed (MEDLINE), and Cochrane Library, from January 2007 to March 2016. Correlated meeting abstracts were also retrieved at the American Society of Clinical Oncology, the European Society for Medical Oncology, and the World Conference of Lung Cancer. The search strategy used both MeSH terms and free-text words to increase sensitivity. A combination of terms was used: "Nivolumab/ONO-4538/BMS-936,558/MDX-1106/Opdivo" and "lung cancer/carcinoma".

\section{Inclusion and exclusion criteria}

Eligible studies were required to meet the following criteria: 1) articles with prospective trials investigating nivolumab on advanced NSCLC patients; 2) studies reporting any of the following information: objective response rate, disease control rate (DCR), OS, progression-free survival (PFS), and grade 3-4 AEs. Exclusion criteria were the following: 1) duplicate publications; 2) studies without usable data; and 3 ) letters, editorials, case reports, and reviews.

\section{Data extraction and quality control}

Our primary outcome was ORR, secondary outcomes PFS, OS, DCR, and toxicity profile with the efficacy dose of $3 \mathrm{mg} / \mathrm{kg}$. Two investigators performed the search independently to avoid bias in the data-extraction process. Disagreement over eligibility of a study was resolved by consensus or by the third investigator. For each study, we extracted the key information: name of the first author, year of publication, trial phase, number of participants, dose, PD-L1 expression level (PD-L1 positive: $\mathrm{PD}-\mathrm{L} 1$ expression $\geq 5 \%$, PD-L1 negative: PD-L1 expression $<5 \%$ ), tumor cell histology as well as the outcomes mentioned above. The data collection and assessment of methodological quality followed the Cochrane Collaboration guidelines (http://www.cochrane.de). In addition, a Newcastle-Ottawa scale was used by three reviewers (J Huang, Y Zhang, and J Sheng) to estimate all the 
prospective and retrospective studies. Discrepancies were discussed by all investigators to reach a consensus. ${ }^{26}$

\section{Statistical analysis}

Odds ratios for dichotomous data (ORR) with 95\% confidence interval (CI) were pooled. Heterogeneity across studies was assessed with a forest plot and the inconsistency statistic $\left(I^{2}\right)$. A random-effects model was employed in case of the existence of potential heterogeneity $\left(I^{2}>50 \%\right)$, otherwise, fixed-effect models were used. All calculations were performed using Review Manager 5.3 (Nordic Cochrane Centre, Copenhagen, Denmark) and R software (version 3.2.2). $P<0.05$ was considered statistically significant for all analyses.

\section{Publication bias}

An extensive search strategy was made to minimize the potential for publication bias. Graphical funnel plots were generated to visually assess a publication bias. Begger's test and Egger's funnel plots were performed to assess the funnel plot asymmetry. 27,28

\section{Results}

\section{Study selection and characteristics}

Overall, a total of 421 records were identified according to the search strategy; 330 records were excluded after screening the titles and abstracts. Fifty-one studies were excluded after reviewing each publication. As for the remaining 13 potentially relevant studies, four studies were excluded due to being without primary outcomes, and finally we enrolled nine studies involving 892 NSCLC patients. ${ }^{13,17,18,20-25}$ Figure 1 shows the studies selection process, and the flow chart of the nine selected clinical trials. Table 1 summarizes the characteristics of the included studies and agents.

\section{Efficacy}

\section{Meta-analysis of nivolumab in terms of ORR, DCR,} $\mathrm{I}$-year-OS rate, and I-year-PFS rate

In summary, at the dose of $3 \mathrm{mg} / \mathrm{kg}$, all trials presented the information on ORR. As shown in Figure 2A, the pooled ORR of the whole population of nivolumab was $20 \%(95 \%$ CI: $17 \%-23 \%)$ without heterogeneity $\left(I^{2}=0 \%, P=0.562\right)$. Only four trials presented the information about DCR, the pooled rate was $36 \%$ (95\% CI: $22 \%-51 \%$ ) with significant heterogeneity $\left(I^{2}=92.4 \%, P<0.0001\right)$, which is presented in Figure 2B. In addition, for the survival benefit, the pooled 1-year-OS rate was $47 \%$ (95\% CI: $40 \%-53 \%$ ) with median heterogeneity $\left(I^{2}=51.3 \%, P=0.103\right.$; Figure $\left.2 \mathrm{C}\right)$, while the

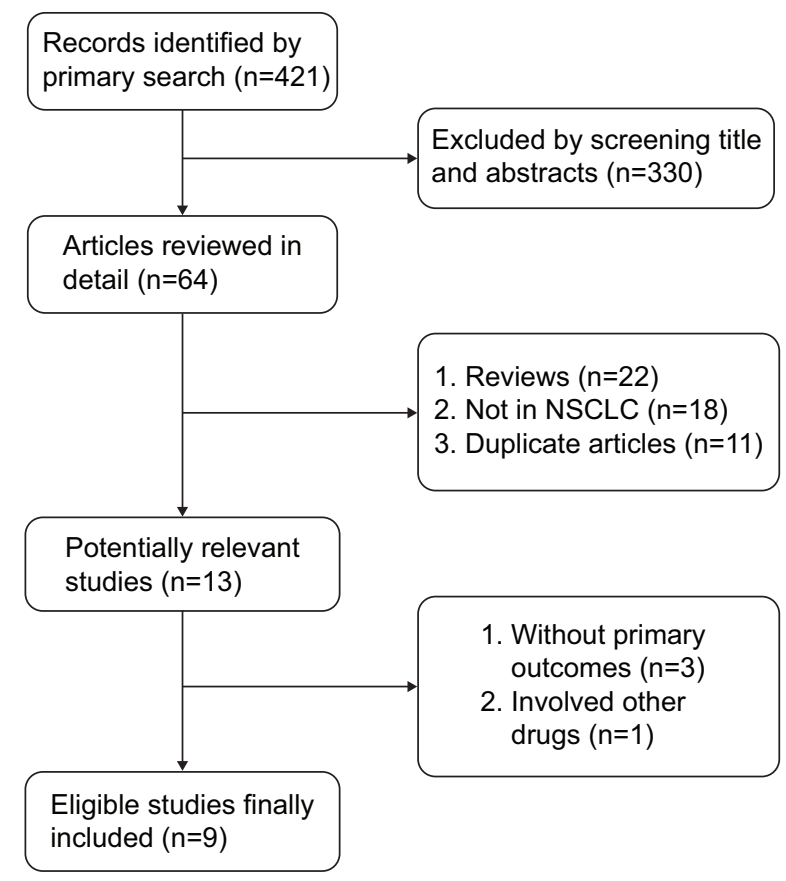

Figure I Flowchart of selecting relevant studies.

pooled 1-year-PFS rate was 21\% (95\% CI: 18\%-24\%) with small heterogeneity $\left(I^{2}=0 \%, P=0.519\right.$; Figure $\left.2 \mathrm{D}\right)$.

\section{Toxicity}

In general, nine studies provided data on grade 3-4 nivolumab-related AEs, while five studies presented the data on any grade AEs. The pooled rate of any grade AEs is $63 \%$ ( $95 \%$ CI: $51 \%-72 \%$ ), while the pooled rate of grade 3-4 AEs is only $8 \%(95 \% \mathrm{CI}: 6 \%-12 \%)$ with significant heterogeneity $\left(I^{2}=75.5 \%, P<0.0001\right)$ (Figure $3 \mathrm{~A}$ and B). Rates of any grade of common AEs of nivolumab were analyzed, and included fatigue (28\%, 95\% CI: $24 \%-33 \%$ ), decreased appetite (13\%, 95\% CI: 9\%-17\%), nausea (12\%, 95\% CI: 9\%-16\%), asthenia (10\%, 95\% CI: $8 \%-13 \%)$, diarrhea (8\%, 95\% CI: 6\%-11\%), pneumonitis (4\%, 95\% CI: $2 \%-6 \%)$, rash $(4 \%, 95 \% \mathrm{CI}$ : $2 \%-6 \%$ ), anemia ( $3 \%, 95 \%$ CI: $1 \%-3 \%$ ), and myalgia ( $3 \%$, 95\% CI: $2 \%-5 \%$ ). However, all common AEs of grade 3-4 were $<10 \%$. More details are presented in Table 2 .

\section{Subgroup analysis}

Non-squamous versus squamous cell lung cancer

As for the different histology types NSCLC, within the efficacy dose of $3 \mathrm{mg} / \mathrm{kg}$. The pooled ORR of squamous cell lung cancer was $20 \%$ (95\% CI: $16 \%-25 \%)$ with small heterogeneity $\left(I^{2}=14.6 \%, P=0.320\right.$ ), while the pooled ORR of non-squamous cell lung cancer was $20 \%$ (95\% CI: 17\%-24\%) with small heterogeneity $\left(I^{2}=0 \%, P=0.888\right)$. Additionally, for 


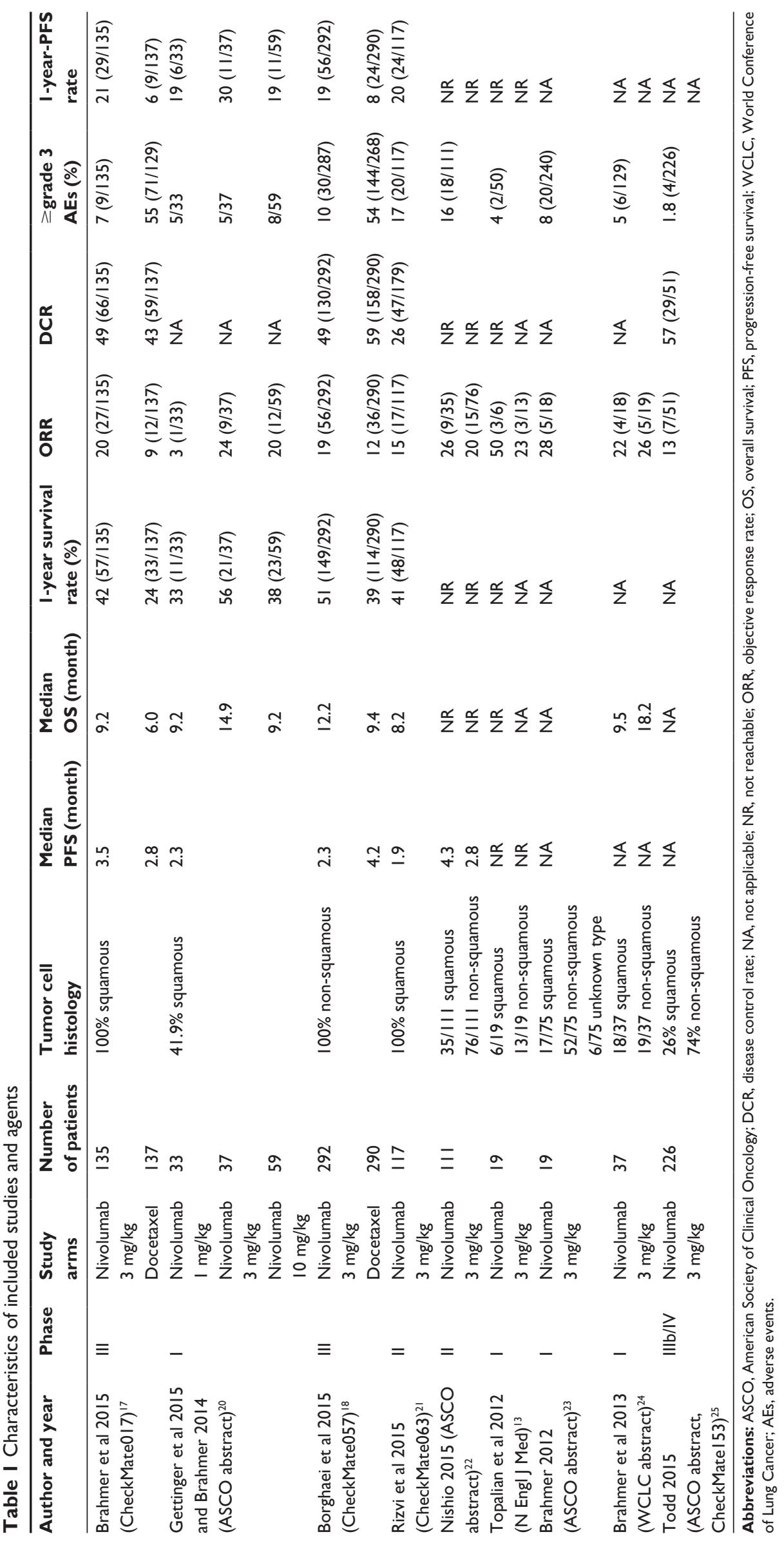




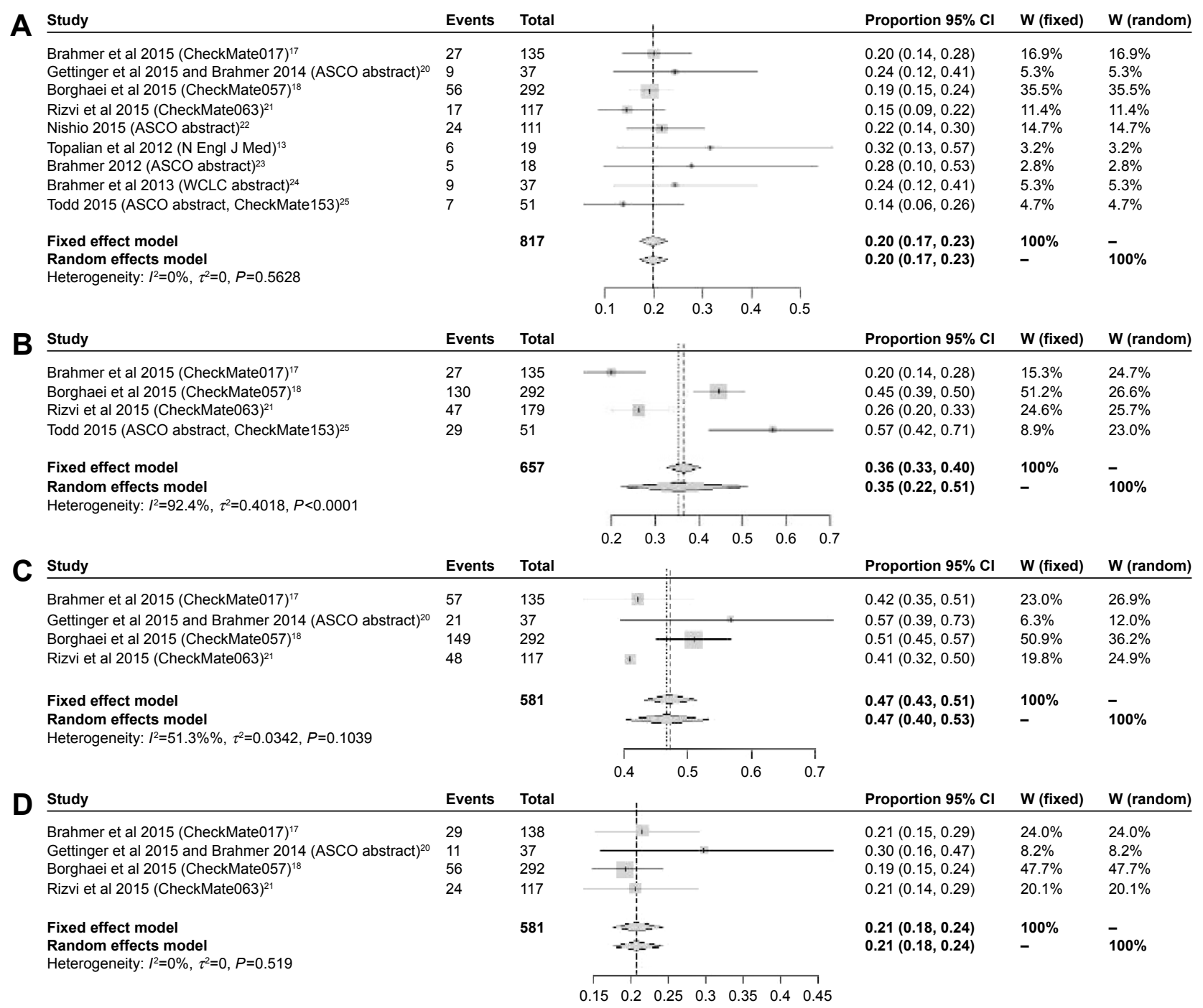

Figure 2 Summary ORR (A), DCR (B), I-year-OS rate (C), I-year-PFS rate (D).

Abbreviations: ASCO, American Society of Clinical Oncology; WCLC, World Conference of Lung Cancer; Cl, confidence interval; DCR, disease control rate; ORR, objective response rate; OS, overall survival; PFS, progression-free survival.

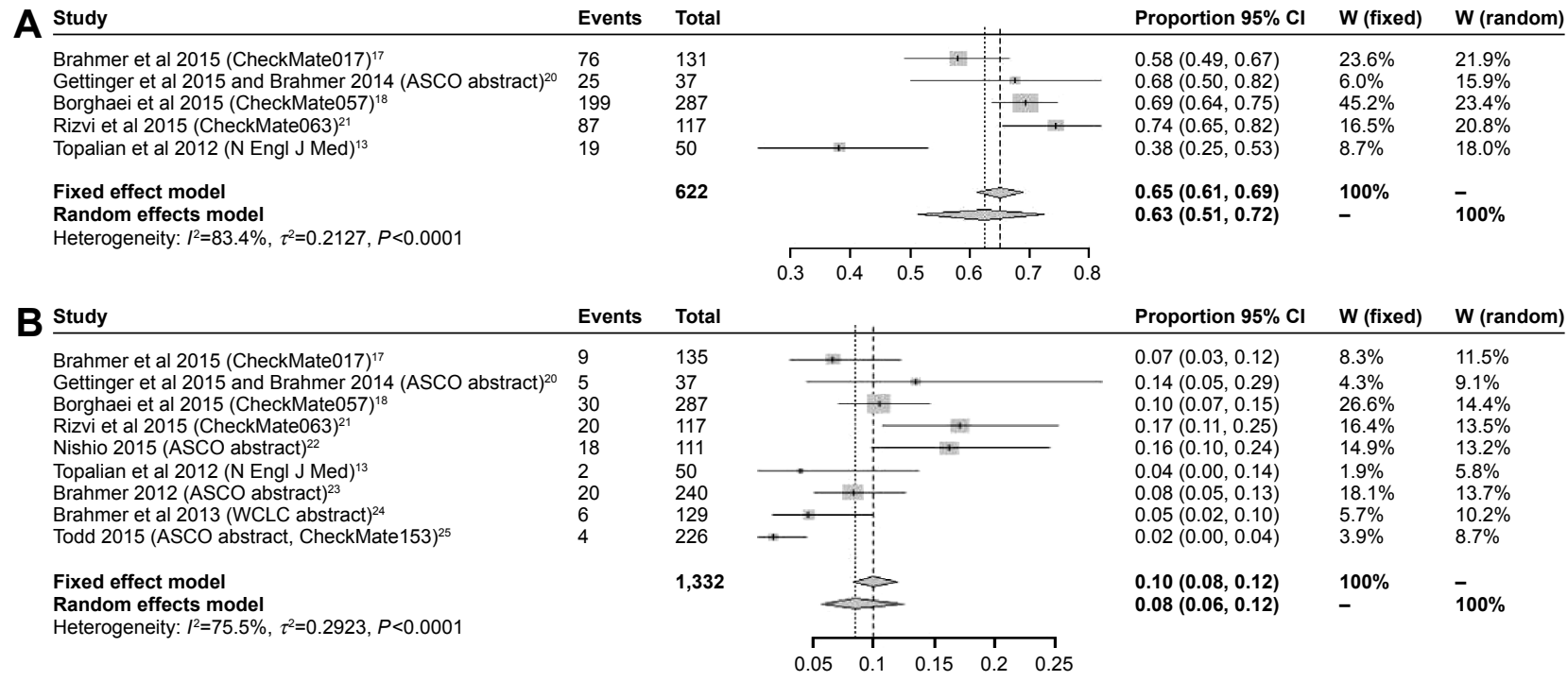

Figure 3 The pooled rate of any grade of AEs of nivolumab $(\mathbf{A})$ and the pooled rate of grade 3-4 AEs of nivolumab (B).

Abbreviations: ASCO, American Society of Clinical Oncology; WCLC, World Conference of Lung Cancer; AE, adverse events; Cl, confidence interval. 
Table 2 Meta-analysis of nivolumab in terms of rate of common AEs

\begin{tabular}{lllll}
\hline $\begin{array}{l}\text { Types of } \\
\text { common AEs }\end{array}$ & $\begin{array}{l}\text { Any grade } \\
\text { rate (\%) }\end{array}$ & $\mathbf{9 5 \%} \mathbf{C l}$ & $\begin{array}{l}\text { Grade 3/4 } \\
\text { rate (\%) }\end{array}$ & $\mathbf{9 5 \%} \mathbf{C l}$ \\
\hline Fatigue & 0.28 & $0.09-0.33$ & 0.08 & $0.06-0.11$ \\
Nausea & 0.12 & $0.09-0.16$ & 0.03 & $0-0.23$ \\
Asthenia & 0.1 & $0.08-0.13$ & 0.02 & $0-0.36$ \\
Anemia & 0.03 & $0.01-0.03$ & 0.01 & $0-0.01$ \\
Decreased appetite & 0.13 & $0.09-0.17$ & 0.01 & $0-0.02$ \\
Myalgia & 0.03 & $0.02-0.05$ & 0.01 & $0-0.02$ \\
Pneumonitis & 0.04 & $0.02-0.06$ & 0.01 & $0-0.03$ \\
Rash & 0.04 & $0.02-0.06$ & 0.01 & $0-0.02$ \\
Diarrhea & 0.08 & $0.06-0.11$ & 0.01 & $0-0.02$ \\
\hline
\end{tabular}

Abbreviations: AEs, adverse effects; $\mathrm{Cl}$, confidence interval.

squamous cell lung cancer patients versus non-squamous cell lung cancer patients treated with nivolumab, the odds ratio of ORR was 1.23 (95\% CI: $0.63-2.39, z=0.61, P=0.54)$ (Figure 4A). Thereby, the ORR of non-squamous cell lung cancer cohort and squamous cell lung cancer cohort had a resemblance.

\section{PD-LI positive versus PD-LI negative}

In respect of PD-L1 expression level (cut-off value $=5 \%$ ), within the efficacy dose of $3 \mathrm{mg} / \mathrm{kg}$. The pooled ORR of PD-L1 positive lung cancer patients was 31\% (95\% CI: $24 \%-38 \%)$ with small heterogeneity $\left(I^{2}=40.1 \%, P=0.188\right)$, while the pooled ORR of PD-L1 negative lung cancer patients was 12\% (95\% CI: 9\%-17\%). Moreover, for PD-L1 positive patients, the odds ratio of ORR for nivolumab was $3.08(95 \%$ CI: 1.87-5.08, $z=4.42, P<0.0001)$, compared to cases with PD-L1 negative tumor (Figure 4B). Therefore, we observed that within the same efficacy dose, greater ORR presented in PD-L1 positive cohort than PD-L1 negative cohort.

\section{Publication bias}

In regard to the publication bias, Egger's funnel plot and Egger's test were performed to assess the publication bias in the literature. The funnel plot and Egger's funnel plot are presented in (Figure 5A and B), respectively. In addition, all nine eligible studies investigating NSCLC patients yielded a Begg's test score of $P=0.3741$ and an Egger's test score of $P=0.3019$. In general, the absence of publication bias was observed for all nine studies, so all these outcomes reveal that there is no significant publication bias.

\section{Discussion}

Nivolumab eliminates cancer cells by attaching to the PD-1 ligand on immune cells, while restoring antitumor immune response and having tolerable AEs. ${ }^{19}$ Nivolumab exhibited survival improvement in naive melanoma at the very start. ${ }^{29}$ Its superior efficacy in melanoma draws enormous attention to investigating the therapeutic effect of nivolumab on NSCLC as well as other tumors. With regard to NSCLC, the efficacy and safety of nivolumab are still under investigation. Although several studies were published, most of them were designed as Phase I or II trials. Although, a series of studies have assessed the efficacy and safety of PD-1 agents, including nivolumab, the efficacy of various doses and safety outcomes of nivolumab were combined, which might lead to huge heterogeneity. ${ }^{30,31}$ So it is important and necessary to merge the data at the same efficacious doses. Therefore,

\begin{tabular}{|c|c|c|c|c|c|}
\hline $\begin{array}{l}\text { Study or } \\
\text { subgroup }\end{array}$ & \multicolumn{2}{|c|}{ Squamous } & \multicolumn{2}{|c|}{ Non-squamous } & $\begin{array}{l}\text { Weight } \\
(\%)\end{array}$ \\
\hline Brahmer et al $2013^{24}$ & 4 & 18 & 5 & 19 & 24.3 \\
\hline Gettinger et al $2015^{20}$ & 4 & 18 & 5 & 19 & 24.3 \\
\hline Nishio $2015^{22}$ & 9 & 35 & 15 & 76 & 45.2 \\
\hline Topalian et al $2012^{13}$ & 3 & 6 & 3 & 13 & 6.1 \\
\hline Total $(95 \% \mathrm{CI})$ & & 77 & & 127 & 100 \\
\hline Total events & 20 & & 28 & & \\
\hline \multicolumn{6}{|c|}{ Heterogeneity: $\chi^{2}=1.61, d f=3(P=0.66) ; l^{2}=0 \%$} \\
\hline \multicolumn{6}{|c|}{ Test for overall effect: $z=0.61(P=0.54)$} \\
\hline
\end{tabular}

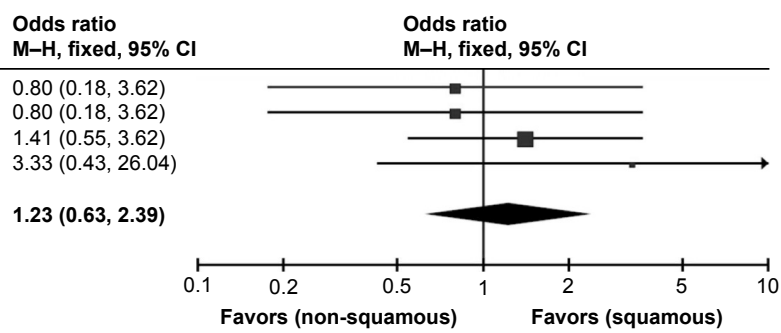

\begin{tabular}{|c|c|c|c|c|c|}
\hline $3 \begin{array}{l}\text { Study or } \\
\text { subgroup }\end{array}$ & $\begin{array}{l}\text { Positive } \\
\text { Events }\end{array}$ & Total & $\begin{array}{l}\text { Negative } \\
\text { Events }\end{array}$ & Total & $\begin{array}{l}\text { Weig } \\
(\%)\end{array}$ \\
\hline Borghaei et al $2015^{18}$ & 34 & 95 & 14 & 136 & 43.2 \\
\hline Brahmer et al $2015^{17}$ & 9 & 42 & 11 & 75 & 36.3 \\
\hline Rizvi et al $2015^{21}$ & 6 & 25 & 7 & 51 & 20.5 \\
\hline Total $(95 \% \mathrm{Cl})$ & & 162 & & 262 & 100 \\
\hline $\begin{array}{l}\text { Total events } \\
\text { Heterogeneity: } \chi^{2}=3.9\end{array}$ & $\begin{array}{l}49 \\
d f=2(P=\end{array}$ & 4); & $\begin{array}{l}32 \\
9 \%\end{array}$ & & \\
\hline
\end{tabular}

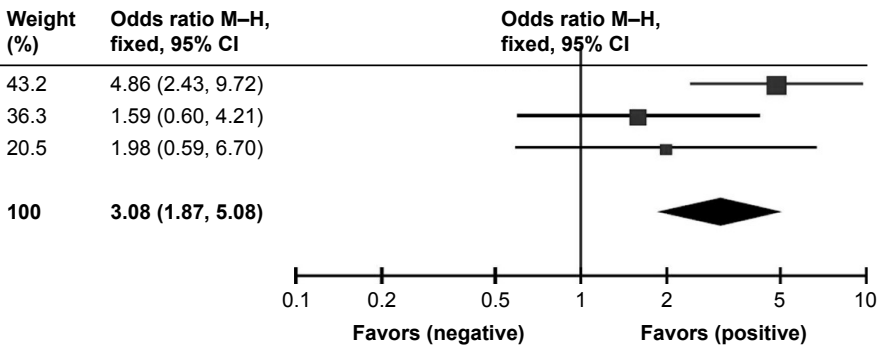

Figure 4 Meta-analysis on ORR of squamous NSCLC versus non-squamous NSCLC (A) and meta-analysis on ORR of PD-LI positive versus PD-LI negative patients (B). Abbreviations: $\mathrm{Cl}$, confidence interval; NSCLC, non-small-cell lung cancer; ORR, objective response rate; PD-LI, programmed cell death ligand I. 


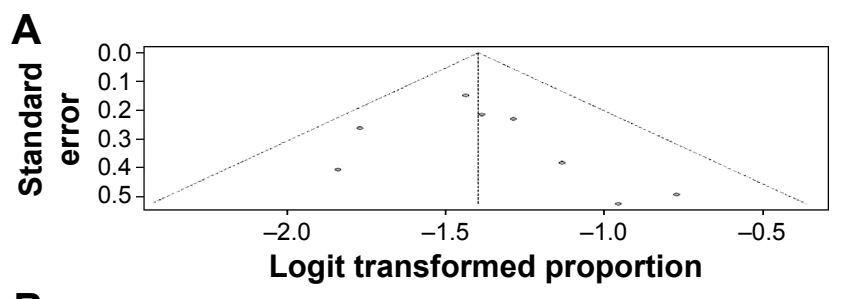

B

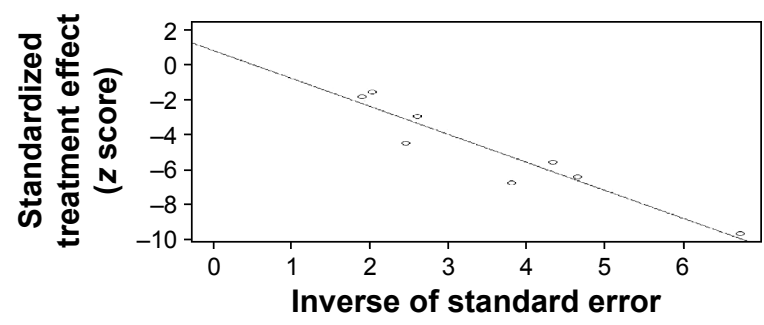

Figure $\mathbf{5}$ The funnel plot of publication bias (A) and the Egger's funnel plot of publication bias (B).

a meta-analysis merging all available data from correlative studies using the standard dose was warranted to show the real clinical efficacy of nivolumab.

In our study, nivolumab showed durable outcome of ORR with tolerable AEs using the dose of $3 \mathrm{mg} / \mathrm{kg}$ in previously treated advanced NSCLC patients. Nevertheless, the ORR of nivolumab was lower than first-line chemotherapy or EGFR tyrosine kinase inhibitors; ${ }^{7,32,33}$ it was higher than second-line standard docetaxel regimen. ${ }^{17,18}$ The other endpoints (DCR,1-year-PFS,1-year-OS) also demonstrated greater efficacy of nivolumab for previously treated advanced NSCLC patients.

Besides, nivolumab revealed manageable toxicities. It was demonstrated that checkpoint inhibitors were associated with a unique series of AEs due to nonspecific immunologic activation. ${ }^{34}$ Recent studies have indicated that these AEs are related to the infiltration of highly activated CD4 and CD8 $\mathrm{T}$-cells and increasing production of inflammatory cytokines in otherwise healthy tissues. ${ }^{35}$ However, we found that the rate of treatment-related AEs of any grade was 63\%, and the grade 3-4 AEs rate was only $8 \%$, which demonstrated that nivolumab is tolerated by these treated patients. The most common treatment-related AEs were fatigue (28\%), decreased appetite (13\%), nausea (12\%), and asthenia (10\%). It will be very important for physicians to notice these immune treatment-related AEs and devise proper strategies to manage these problems. As a result, nivolumab is tolerable and regarded as a second-line regimen for previously treated advanced NSCLC patients.

As for subgroup analyses, we demonstrated that using the standard dose of $3 \mathrm{mg} / \mathrm{kg}$, the pooled ORR of squamous NSCLC was similar to non-squamous NSCLC, which reconfirmed clinical efficacy with nivolumab in both squamous and non-squamous NSCLC patients after initial treatment failures. In addition, regardless of the histological types, the efficacy of nivolumab was higher than standard second-line regimen with tolerable AEs. ${ }^{9,10}$ In respect of PD-L1 status (cut-off value $=5 \%$ ), we clarified that the ORR in PD-L1 positive cohort was higher than PD-L1 negative cohort. This finding was in accordance with previous studies. ${ }^{13,36}$ It seems that high-level PD-L1 expression might be a promising response predictor for nivolumab treatment. However, there are several limitations in the study. Most of the included studies were Phase I trials, and there was a lack of sufficient data and sample size to be reliable. Moreover, on the basis of our study, the efficacy and safety of nivolumab combining with other therapy was unknown. Further studies are needed to resolve these limitations.

In conclusion, nivolumab is a promising second-line agent for previously treated advanced NSCLC with manageable AEs. Both squamous and non-squamous NSCLC patients showed similar efficacy. In addition, patients with positive PD-L1 had a higher response rate to nivolumab.

\section{Acknowledgments}

No funding was involved. Jiaxing Huang, Yaxiong Zhang, and Jin Sheng share first authorship.

\section{Disclosure}

The authors report no conflicts of interest in this work.

\section{References}

1. Siegel RL, Miller KD, Jemal A. Cancer statistics, 2016. CA Cancer J Clin. 2016;66(1):7-30.

2. Parums DV. Current status of targeted therapy in non-small cell lung cancer. Drugs Today (Barc). 2014;50(7):503-525.

3. DeSantis CE, Lin CC, Mariotto AB, et al. Cancer treatment and survivorship statistics, 2014. CA Cancer J Clin. 2014;64(4):252-271.

4. Guilbert JJ. The world health report 2002 - reducing risks, promoting healthy life. Educ Health (Abingdon). 2003;16(2):230.

5. Jackman DM, Miller VA, Cioffredi LA, et al. Impact of epidermal growth factor receptor and KRAS mutations on clinical outcomes in previously untreated non-small cell lung cancer patients: results of an online tumor registry of clinical trials. Clin Cancer Res. 2009;15(16): $5267-5273$

6. D'Addario G, Fruh M, Reck M, Baumann P, Klepetko W, Felip E; ESMO Guidelines Working Group. Metastatic non-small-cell lung cancer: ESMO Clinical Practice Guidelines for diagnosis, treatment and follow-up. Ann Oncol. 2010;21(Suppl 5):v116-v119.

7. Zhou C, Wu YL, Chen G, et al. Erlotinib versus chemotherapy as first-line treatment for patients with advanced EGFR mutation-positive non-small-cell lung cancer (OPTIMAL, CTONG-0802): a multicentre, open-label, randomised, phase 3 study. Lancet Oncol. 2011;12(8) 735-742.

8. Cufer T, Ovcaricek T, O'Brien ME. Systemic therapy of advanced non-small cell lung cancer: major-developments of the last 5-years. Eur J Cancer. 2013;49(6):1216-1225. 
9. Shepherd FA, Dancey J, Ramlau R, et al. Prospective randomized trial of docetaxel versus best supportive care in patients with non-small-cell lung cancer previously treated with platinum-based chemotherapy. J Clin Oncol. 2000;18(10):2095-2103.

10. Fossella FV, DeVore R, Kerr RN, et al. Randomized phase III trial of docetaxel versus vinorelbine or ifosfamide in patients with advanced non-small-cell lung cancer previously treated with platinum-containing chemotherapy regimens. The TAX 320 Non-Small Cell Lung Cancer Study Group. J Clin Oncol. 2000;18(12):2354-2362.

11. Korman AJ, Peggs KS, Allison JP. Checkpoint blockade in cancer immunotherapy. Adv Immunol. 2006;90:297-339.

12. Pardoll DM. The blockade of immune checkpoints in cancer immunotherapy. Nat Rev Cancer. 2012;12(4):252-264.

13. Topalian SL, Hodi FS, Brahmer JR, et al. Safety, activity, and immune correlates of anti-PD-1 antibody in cancer. N Engl J Med. 2012;366(26): 2443-2454.

14. Wang $\mathrm{C}$, Thudium KB, Han $\mathrm{M}$, et al. In vitro characterization of the anti-PD-1 antibody nivolumab, BMS-936558, and in vivo toxicology in non-human primates. Cancer Immunol Res. 2014;2(9):846-856.

15. Brahmer JR, Drake CG, Wollner I, et al. Phase I study of single-agent anti-programmed death-1 (MDX-1106) in refractory solid tumors: safety, clinical activity, pharmacodynamics, and immunologic correlates. J Clin Oncol. 2010;28(19):3167-3175.

16. Haanen JB, Thienen H, Blank CU. Toxicity patterns with immunomodulating antibodies and their combinations. Semin Oncol. 2015;42(3): 423-428.

17. Brahmer J, Reckamp KL, Baas P, et al. Nivolumab versus docetaxel in advanced squamous-cell non-small-cell lung cancer. $N$ Engl J Med. 2015;373(2):123-135.

18. Borghaei H, Paz-Ares L, Horn L, et al. Nivolumab versus docetaxel in advanced nonsquamous non-small-cell lung cancer. $N$ Engl J Med. 2015;373(17):1627-1639.

19. Dizon DS, Krilov L, Cohen E, et al. Clinical cancer advances 2016: annual report on progress against cancer from the American Society of Clinical Oncology. J Clin Oncol. 2016;34(9):987-1011.

20. Gettinger SN, Horn L, Gandhi L, et al. Overall survival and long-term safety of nivolumab (Anti-programmed Death 1 Antibody, BMS936558, ONO-4538) in patients with previously treated advanced non-small-cell lung cancer. J Clin Oncol. 2015;33(18):2004-2012.

21. Rizvi NA, Mazieres J, Planchard D, et al. Activity and safety of nivolumab, an anti-PD-1 immune checkpoint inhibitor, for patients with advanced, refractory squamous non-small-cell lung cancer (CheckMate 063): a phase 2, single-arm trial. Lancet Oncol. 2015;16(3):257-265.

22. Makoto Nishio THKN. Phase II studies of nivolumab (anti-PD-1, BMS-936558, ONO-4538) in patients with advanced squamous (sq) or nonsquamous (non-sq) non-small cell lung cancer (NSCLC). J Clin Oncol. 2015;33(20 Suppl):8027.
23. Julie R, Brahmer LHSA. Clinical activity and safety of anti-PD1 (BMS936558, MDX-1106) in patients with advanced non-small-cell lung cancer (NSCLC). In: ASCO Annual Meeting. 2012(suppl; abstr):7509.

24. Julie R, Brahmer LHSJ, Leena Gandhi LVSV, Ahlers JMWG, Gettinger. Nivolumab (anti-PD-1; BMS-936558; ONO-4538) in patients with non-small cell lung cancer (NSCLC): overall survival and long-term safety in a phase 1 trial. In: WCLC Annual Meeting. 2013:909.

25. Todd Michael Bauer MMJC. An ongoing phase IIIb/IV safety trial of nivolumab (NIVO) in patients (pts) with advanced or metastatic nonsmall-cell lung cancer (NSCLC) who progressed after receiving 1 or more prior systemic regimens. 2015 ASCO Annual Meeting; 2015.

26. Wells G, Shea B, O'Connell D, et al. The Newcastle-Ottawa Scale (NOS) for assessing the quality of nonrandomized studies in meta-analyses. Department of Epidemiology and Community Medicine, University of Ottawa, Canada. Available from: http://www.ohri.ca/programs/ clinical_epidemiology/oxford.asp

27. Begg CB, Mazumdar M. Operating characteristics of a rank correlation test for publication bias. Biometrics. 1994;50(4):1088-1101.

28. Egger M, Davey SG, Schneider M, Minder C. Bias in meta-analysis detected by a simple, graphical test. BMJ. 1997;315(7109):629-634.

29. Robert C, Long GV, Brady B, et al. Nivolumab in previously untreated melanoma without BRAF mutation. $N$ Engl J Med. 2015;372(4): 320-330.

30. Jia M, Feng W, Kang S, et al. Evaluation of the efficacy and safety of anti-PD-1 and anti-PD-L1 antibody in the treatment of non-small cell lung cancer (NSCLC): a meta-analysis. J Thorac Dis. 2015;7(3): $455-461$.

31. Zhu L, Jing S, Wang B, Wu K, Shenglin MA, Zhang S. Anti-PD-1/ PD-L1 therapy as a promising option for non-small cell lung cancer: a single arm meta-analysis. Pathol Oncol Res. 2016;22(2):331-339.

32. Lynch TJ, Bell DW, Sordella R, et al. Activating mutations in the epidermal growth factor receptor underlying responsiveness of non-small-cell lung cancer to gefitinib. N Engl J Med. 2004;350(21):2129-2139.

33. Rossi A, Pasquale R, Esposito C, Normanno N. Should epidermal growth factor receptor tyrosine kinase inhibitors be considered ideal drugs for the treatment of selected advanced non-small cell lung cancer patients? Cancer Treat Rev. 2013;39(5):489-497.

34. Postow MA, Callahan MK, Wolchok JD. Immune checkpoint blockade in cancer therapy. J Clin Oncol. 2015;33(17):1974-1982.

35. Kaehler KC, Piel S, Livingstone E, Schilling B, Hauschild A, Schadendorf D. Update on immunologic therapy with anti-CTLA-4 antibodies in melanoma: identification of clinical and biological response patterns, immune-related adverse events, and their management. Semin Oncol. 2010;37(5):485-498.

36. Taube JM, Klein A, Brahmer JR, et al. Association of PD-1, PD-1 ligands, and other features of the tumor immune microenvironment with response to anti-PD-1 therapy. Clin Cancer Res. 2014;20(19):5064-5074.
OncoTargets and Therapy

\section{Publish your work in this journal}

OncoTargets and Therapy is an international, peer-reviewed, open access journal focusing on the pathological basis of all cancers, potential targets for therapy and treatment protocols employed to improve the management of cancer patients. The journal also focuses on the impact of management programs and new therapeutic agents and protocols on
Dovepress

patient perspectives such as quality of life, adherence and satisfaction The manuscript management system is completely online and includes a very quick and fair peer-review system, which is all easy to use. Visit http://www.dovepress.com/testimonials.php to read real quotes from published authors. 